\title{
La mujer: pieza clave en el desarrollo sostenible. Estrategias contenidas en la Agenda 2030
}

\author{
Women: a key element in sustainable development. \\ Strategies contained in the 2030 Agenda
}

\begin{abstract}
Resumen
Desde hace poco más de 50 años que se viene considerando la importancia de incorporar a la mujer en las estrategias para el desarrollo. Durante los años setenta surgió el enfoque de mujeres para el desarrollo (MED) para fomentar el acceso de las mujeres a los recursos productivos y con ello promover la igualdad de género. Posteriormente, encontramos juntos por primera vez los conceptos de mujer y desarrollo sostenible en la Cumbre de la Tierra llevada a cabo en Río de Janeiro en 1992. Desde entonces se ha continuado trabajando en la incorporación del enfoque de género en la agenda internacional para la promoción del desarrollo sostenible, tal como se refleja tanto en la Declaración del Milenio como en la Agenda 2030. Por ello, este trabajo tiene como objetivo analizar la importancia de la participación activa de la mujer tanto en la Agenda 2030 para el desarrollo sostenible como en la promoción de una cultura de desarrollo sostenible con perspectiva de género, destacando, finalmente, que tras más de 50 años de trabajo continuado siguen existiendo grandes retos para que se produzca una plena incorporación. Todo ello bajo una metodología descriptiva basada en el análisis de datos cualitativos, sobre la bibliografía existente en la materia, y cuantitativos sobre el análisis de los indicadores de género contenidos en la Agenda 2030.
\end{abstract}

Palabras clave: enfoque y perspectiva de género, indicadores de género en el desarrollo sostenible,Agenda 2030, cultura de género, derechos de la mujer.

\section{Diana Marcela Verdiales López*}

\begin{abstract}
For just over 50 years, the importance of incorporating women into development strategies has been considered. During the 1970s, theWomen's Approach to Development (MED) emerged to promote women's access to productive resources and thereby promote gender equality. Subsequently, we found together for the first time the concepts of Women and Sustainable Development at the Earth Summit held in Rio de Janeiro in 1992. Since then, work has continued on the incorporation of the gender approach in the international agenda for the promotion sustainable development, as reflected in both the Millennium Declaration and the 2030 Agenda. Therefore, this work aims to analyze the importance of the active participation of women both in the 2030 Agenda for sustainable development and in the promotion of a culture of sustainable development with a gender perspective, highlighting, finally, that after more than 50 years of continuous work, there are still great challenges for full incorporation to take place. All this under a descriptive methodology based on the analysis of qualitative data, on the existing bibliography on the matter, and quantitative on the analysis of the gender indicators contained in the 2030 Agenda.
\end{abstract}

Keywords: gender perspective and approach, gender indicators in sustainable development, 2030 Agenda, gender culture, women's rights.

-Doctora en Derechos Humanos, Paz y Desarrollo Sostenible por la Universidad de Valencia. Docente-Investigadora y Responsable de Proyectos de Investigación y Cooperación al Desarrollo en el Centro de Estudios de Iberoamérica de la Universidad Rey Juan Carlos_diana.verdiales@urjc.es_ORCID: 0000-0003-3275-2058

Fecha de recepción: 14 de abril de 2020. Fecha de aceptación: 25 de junio de 2021 . 


\section{Introducción}

La participación de la mujer en las cuestiones relativas al desarrollo tuvo sus orígenes en los años setenta con la construcción del enfoque de mujeres en el desarrollo (MED), donde se pusieron de manifiesto las desigualdades existentes entre hombres y mujeres. Este enfoque pretende representar a las mujeres como miembros productivos en la sociedad, facilitando los recursos productivos para promover la igualdad, sin tomar en consideración las relaciones de poder, de socialización y de género. ${ }^{1}$ Posteriormente se desarrollaron los enfoques de género en el desarrollo (GED) y el enfoque de género (mainstreaming). El primero fue desarrollado durante los años ochenta y pretendía transformar las relaciones de poder desiguales que obstaculizan el disfrute equitativo de las mujeres en todos los ámbitos del desarrollo. ${ }^{2}$ El segundo, por su parte, se desarrolló entre los años noventa y principios del año 2000 para identificar y tomar en consideración las necesidades que afectan tanto a los hombres como a las mujeres en el desarrollo. ${ }^{3}$

Después de más de 50 años de trabajo continuado, por parte de diversos organismos internacionales para que se reconozcan plenamente los derechos de todas las mujeres y niñas a nivel mundial, siguen existiendo obstáculos de diversa índole que impiden su plena participación en todas las esferas de la sociedad. Desde la Carta de las Naciones Unidas (1945), la Comisión de la Condición Jurídica y Social de la Mujer (1946), la Convención sobre la Eliminación de Todas las Formas de Discriminación Contra la Mujer

I. Fernández Saavedra Ana G., y Dema Moreno, Sandra. (2018). La integración de la perspectiva de género en la gestión del riesgo de desastres: De los ODM a los ods. Revista Internacional de Cooperación y Desarrollo, 5(I): 33.

2. Ministerio de Asuntos Exteriores y de Cooperación. (2007). Estrategia de "género en desarrollo" de la cooperación española (pp. 33 y 34).

3. Fundación Mujeres. (2003). Guía para la elaboración de proyectos desde una perspectiva de género (p.43). Fondo Social Europeo y Gobierno del Principado de Asturias. 
(1979), la Conferencia Mundial del Decenio de las Naciones Unidas para la Mujer en Copenhague (1980), la Conferencia Mundial para el Examen y la Evaluación de los logros del Decenio de las Naciones Unidas para la Mujer en Nairobi (1985), la Declaración de Río (1992), la Conferencia Mundial de Derechos Humanos de Viena (1993), la Declaración y Plataforma de Acción de Beijing de la Cuarta Conferencia Mundial sobre la Mujer (1995), la Conferencia Internacional sobre Población y Desarrollo en el Cairo (1994), la Cumbre Mundial sobre Desarrollo Social en Copenhague (1995), la Cuarta Conferencia Mundial sobre la Mujer en Beijing (1995), el Acuerdo ACP-CE y la Declaración del Milenio (2000) las Agendas de la Eficacia de la Ayuda de París (2005), Accra (2008) y Busán (2011), el Plan de Acción para la Igualdad de Género y el Empoderamiento de las Mujeres en el Desarrollo (2010-2015) y más recientemente la Agenda 2030 para el desarrollo sostenible (2015), se ha puesto de manifiesto el compromiso de la sociedad internacional para trabajar conjuntamente por la igualdad de género y el empoderamiento de la mujer bajo un sólido marco legislativo e institucional aplicable en todo el mundo y sobre todo resaltar el papel de la mujer como pieza clave para el desarrollo sostenible.

La primera vez que aparecieron juntos los conceptos mujer y desarrollo fue en 1979 en la promulgación de la Convención sobre la Eliminación de Todas las Formas de Discriminación contra la Mujer. Dicha Convención hace referencia al principio de la no discriminación emanado de la Declaración Universal de los Derechos Humanos y, específicamente, a la discriminación contra la mujer ya que ésta viola los principios de la igualdad de derechos y del respeto de la dignidad humana. Asimismo, enfatiza que dicha discriminación por cuestiones de género "constituye un obstáculo para el aumento del bienestar de la sociedad y de la familia y que entorpece el pleno desarrollo de las posibilidades de la mujer para prestar servicio a su país y 
a la humanidad". ${ }^{4}$ Posteriormente, fue en la Cumbre de la Tierra de Río de Janeiro, Brasil (1992) ${ }^{5}$ donde se vinculó a la mujer con el desarrollo sostenible. La Declaración emanada de dicha Cumbre pone de manifesto en el Principio 20 que "Las mujeres desempeñan un papel fundamental en la ordenación del medio ambiente y en el desarrollo. Es, por tanto, imprescindible contar con su plena participación para lograr el desarrollo sostenible". ${ }^{6}$

Posteriormente, en la Declaración derivada de la Conferencia Mundial de Derechos Humanos en Viena (1993) se pone de manifesto en el artículo 18 que:

Los derechos humanos de la mujer y de la niña son parte inalienable, integrante e indivisible de los derechos humanos universales. La plena participación, en condiciones de igualdad, de la mujer en la vida política, civil, económica, social y cultural en los planos nacional, regional e internacional y la erradicación de todas las formas de discriminación basadas en el sexo son objetivos prioritarios de la comunidad internacional.7

Por su parte, la Cuarta Conferencia Mundial sobre la Mujer y Plataforma de Acción de Beijing, China (1995) promueven acciones bajo el lema "el empoderamiento de la mujer empodera a la humanidad" para continuar trabajando en la promoción de diversas acciones hasta lograr la igualdad de género, el empoderamiento de las mujeres y el reconocimiento de los derechos de todas la mujeres y niñas en el mundo, como principal hoja de ruta para combatir contra los

4. Asamblea General de las Naciones Unidas. (1979). Convención sobre la Eliminación de Todas las Formas de Discriminación Contra la Mujer (p. I).

5. Cf. en: https://www.mapa.gob.es/ministerio/pags/biblioteca/revistas/pdf_drs/ drs_4_28_29.pdf

6. Véase en: https://www.un.org/spanish/esa/sustdev/agenda2 I/riodeclaration.htm 7. Cf. en: https://documents-dds-ny.un.org/doc/undoc/gen/g93//42/36/pdf/ g93 I4236.pdf?openelement 
obstáculos y restricciones que impiden el empoderamiento de las mujeres en todo el mundo. ${ }^{8}$

Asimismo, desde las Naciones Unidas se manifestó el compromiso de continuar trabajando por una sociedad más justa y equitativa promoviendo los Objetivos de Desarrollo del Milenio (oDM, 2000-2015) plasmados en la Declaración del Milenio, y los Objetivos de Desarrollo Sostenible (oDs, 2015-2030) en la Agenda 2030, bajo el lema "que nadie se quede atrás". ${ }^{9}$ En ambos Objetivos (ODM y ODs) se ha incluido la perspectiva de género en menor y mayor medida. En los oDM se incluyó la participación activa de la mujer en el Objetivo 3: "Promover la igualdad entre los géneros y la autonomía de la mujer", para trabajar por la igualdad entre géneros y el empoderamiento y la autonomía de la mujer, buscando la eliminación de las desigualdades. En los ODS, de una forma más ampliada esta cuestión queda contenida en el oDs 5: "Igualdad de género", aunque transversalmente podemos ver cómo se ha incluido la perspectiva de género en 10 Objetivos más, como veremos con detalle más adelante. Asimismo, se abordará la importancia de trabajar hacia la construcción de una cultura de desarrollo sostenible con perspectiva de género, y finalmente se abordarán los principales retos existentes para lograr la plena participación de las mujeres en las diversas esferas del desarrollo sostenible.

\section{La necesaria incorporación del enfoque de género en la Agenda 2030}

La urgente necesidad de incorporar la perspectiva de género en la agenda internacional viene apoyada por el deterioro de las condiciones de vida de muchas mujeres

8. Verdiales López, D. (2020). La importancia de la mujer en el desarrollo.Análisis de los Objetivos de Desarrollo Sostenible con perspectiva de género (p. 99).

9. Asamblea General de Naciones Unidas. (2015). Transformar nuestro mundo: La Agenda 2030 para el Desarrollo Sostenible. Resolución 70/I, 25 de septiembre de 2015 , p. 13. 
alrededor del mundo, por las vulnerabilidades que estas condiciones acarrean y por la creciente desigualdad que aqueja cada vez más en la mayoría de los países del mundo. Por ello es importante señalar que, aunque los primeros estudios sobre género se originaron a mediados del siglo XIX, los avances en la materia no han logrado mejorar las condiciones de vida de la mayoría de las mujeres en el mundo. ${ }^{10} \mathrm{En}$ concreto, el concepto de género como bien señala Calatrava, es un concepto relacionado con las relaciones socioeconómicas y culturales entre personas de diferente sexo. ${ }^{11}$ Esto tiene relación con lo que social y culturalmente se espera de nosotros por la manera en la que nos relacionamos en función de nuestro sexo, estereotipos, roles y responsabilidades por el hecho de ser mujer u hombre. ${ }^{12}$

La importancia de incorporar el enfoque de género en la agenda internacional tiene sus orígenes en la definición que hizo el Consejo Económico y Social (Ecosoc 1997/2) sobre dicha iniciativa como

[...] una estrategia para hacer que las preocupaciones y experiencias de mujeres y hombres sean una dimensión integral del diseño, implementación, monitoreo y evaluación de políticas y programas en todas las esferas políticas, económicas y sociales para que las mujeres y los hombres se beneficien por igual,y la desigualdad no se perpetre. Tanto las intervenciones especíicas como las operaciones integradas son formas válidas de medidas de incorporación de la perspectiva de género. ${ }^{13}$

10. Verdiales López, D. (2020). La importancia de la mujer en el desarrollo.Análisis de los Objetivos de Desarrollo Sostenible con perspectiva de género (p. 100).

II. Calatrava, J. (2002). Mujer y desarrollo rural en la globalización: De los proyectos asistenciales a la planificación de género. Revista Globalización y Mundo Rural, núm. 803, p. 73.

I2. Cf. AECID (20|5). Guía de la aecid para la transversalización del enfoque de género (p. 14).

13. Grupo de las Naciones Unidas para el Desarrollo Sostenible.Véase en:https:// unsdg.un.org/es/2030-agenda/universal-values/gender-equality-and-womens-empowerment 
En este sentido, dentro del Sistema de Naciones Unidas se crearon dos mecanismos de responsabilidad compartida para la promoción de la igualdad de género y el empoderamiento de las mujeres y niñas: el Plan de Acción ONU-SWAP 2.0 (2012) y el Cuadro de indicadores de desempeño (2018). El Plan de Acción ONU-SWAP 2.0 ${ }^{14}$ pretende proporcionar un marco de rendición de cuentas clasificado en cinco niveles para autoevaluar e informar sobre la posición de cada indicador respecto al compromiso adquirido en materia de igualdad de género y empoderamiento de las mujeres y avanzar progresivamente hacia el total cumplimiento de los indicadores; y el Cuadro de indicadores de desempeño $(2018)^{15}$ funge como una hoja de ruta o autoevaluación estandarizada de las prácticas y desempeño que deben desarrollar los países para apoyar la incorporación de la perspectiva de género en los planes y acciones nacionales.

Estas acciones se apoyan en el Marco de Cooperación de las Naciones Unidas para el Desarrollo Sostenible (2019). ${ }^{16}$ Este Marco de actuación tiene como propósito ser el instrumento más importante para la planificación y ejecución de las actividades relacionadas con la implementación de la Agenda 2030. Tanto el enfoque basado en los derechos humanos (EBDH) como la igualdad de género y el empoderamiento de las mujeres son algunos de los seis principios rectores del Marco de Cooperación de las Naciones Unidas

14. Onu Mujeres. (20I2). UN System-wide action plan for implementation of the ceb United Nations System wide policy on Gender Equal and the empowerment of women. https://www.unwomen.org/-/media/headquarters/attachments/sections/how\%20 we\%20work/unsystemcoordination/un-swap/un-swap-framework-dec-20I 2. pdf?la=es\&vs $=1815$

I5. Cf. en: https://unsdg.un.org/es/2030-agenda/universal-values/gender-equalityand-womens-empowerment

16. Creado en 2019 mediante la resolución $72 / 279$ de la Asamblea General de las Naciones Unidas y denominado Marco de Asistencia de las Naciones Unidas para el Desarrollo, actualmente llamado Marco de Cooperación de las Naciones Unidas para el Desarrollo Sostenible. Grupo de las Naciones Unidas para el Desarrollo Sostenible. (2019). Marco de Cooperación de las Naciones Unidas para el Desarrollo Sostenible. Directrices internas. Naciones Unidas. 
para el Desarrollo Sostenible, exhortando a los Equipos de país de las Naciones Unidas (UNCT, por sus siglas en inglés) a integrar el enfoque de género en los planes y programas nacionales orientados en el proceso de cooperación.

Como bien señala Machado López et al., para el Grupo de las Naciones Unidas para el Desarrollo Sostenible la desigualdad de género no es sólo una cuestión moral y social apremiante, sino un desafío económico importante. Ya que reconoce que las mujeres constituyen más del $50 \%$ de la población mundial, representando una gran fuerza creativa y productiva en los diversos sectores de la sociedad, tanto políticos como económicos y socioculturales. ${ }^{17}$

Por su parte, el Consejo Económico y Social de Naciones Unidas enfatiza que la transversalización del enfoque de género es

[...] el proceso de evaluar las implicaciones para mujeres y hombres de cada acción planificada, incluida la legislación, políticas o programas, en todas las áreas y a todos los niveles; [se trata pues de] [...] una estrategia para hacer de todas la preocupaciones y experiencias, tanto de las mujeres como de los hombres, una dimensión integral del diseño, la implementación, el seguimiento y la evaluación de las políticas y programas en todas la esferas políticas, económicas y sociales de forma que las mujeres $y$ los hombres se beneficien igualmente y la desigualdad no se perpetúe, [teniendo como objetivo principal el] conseguir la igualdad de género. ${ }^{18}$

De acuerdo con lo que bien señala Marcela Lagarde, la incorporación del enfoque con perspectiva de género es una cuestión clave para el logro de los ODS y por ende del logro

17. Machado López, L., Morales Molina, T., y Chávez Calle, L. S. (2018). La igualdad de género, paradigma del desarrollo sostenible en la Agenda 2030. Revista Universidad y Sociedad.

18. Zabala, l., et al. (20I2).Análisis de la integración del enfoque de género en las políticas de cooperación al desarrollo: El caso de las instituciones de la Comunidad Autónoma del País Vasco. Estudios de Economía Aplicada, pp. 944 y 945. 
del desarrollo humano. Ya que el enfoque con perspectiva de género pretende

[...] enfocar, analizar y comprender las características que definen a mujeres y hombres de manera específica, así como sus semejanzas y diferencias, [analizando] [...] las posibilidades vitales de unas y otros, el sentido de sus vidas, sus expectativas y oportunidades, así como poder identificar [...] los conflictos institucionales y cotidianos que deben encarar y los múltiples modos en que lo hacen. ${ }^{19}$

Por tanto, resulta impensable que se pueda lograr un desarrollo sostenible sin la activa participación de la mujer en las diversas esferas de la sociedad. Es por ello que es de gran importancia continuar trabajando en las acciones emprendidas para incorporar diversos enfoques que promuevan la igualdad de género y el empoderamiento de las mujeres y las niñas en todo el mundo. Estos enfoques son claves en los procesos de implementación de los Objetivos de Desarrollo Sostenible, ya que no sólo contribuyen a la visión transversal del enfoque de género en cada uno de los 17 oDs, sino que también proporcionan una visión más completa sobre las estrategias requeridas para alcanzar un desarrollo humano sostenible. ${ }^{20}$

Recordemos que en la actualidad en muchos países se siguen aplicando prácticas discriminatorias contra la mujer, sobre todo aquellas relacionadas con la salud, la nacionalidad, la herencia o la familia; que siguen recayendo en ellas mayoritariamente las tareas de cuidados en el hogar y las labores domésticas, las cuales les imposibilitan su incor-

19. Lagarde, M. (1996). Género y feminismo. Desarrollo humano y democracia. Madrid:Horas Horas;y en Machado López, L., Morales Molina,T.,y Chávez Calle, L. S. (20I8). La igualdad de género, paradigma del desarrollo sostenible en la Agenda 2030. Revista Universidad y Sociedad, p. 8.

20. Verdiales López, Diana M. (2020). La importancia de la mujer en el desarrollo. Análisis de los Objetivos de Desarrollo Sostenible con perspectiva de género. Femeris: Revista Multidisciplinar de Estudios de Género. 
poración plena en el mercado laboral; que sigue existiendo una brecha salarial de alrededor del $25 \%$ a nivel mundial; y que la pobreza afecta más a las mujeres que a los hombres. Éstas y muchas otras cuestiones hacen necesaria, urgente e imprescindible la incorporación de enfoques que promuevan la igualdad de género y el empoderamiento de las niñas y las mujeres alrededor del mundo.

En este sentido, para apoyar la incorporación del enfoque de género en los oDs la Comisión de la Condición Jurídica y Social de la Mujer ${ }^{21}$ aprobó en el $60^{\circ}$ periodo de sesiones en 2016 una hoja de ruta que facilita la incorporación de la perspectiva de género en la implementación de los ODS, al tiempo que se trabajara en la evaluación de los resultados y los avances logrados en materia de igualdad y de empoderamiento de la mujer.

$\mathrm{Al}$ margen del tipo de enfoque que se quiera implementar para la promoción de la igualdad de género, debemos de tener en cuenta ciertos principios básicos propuestos por la División de las Naciones Unidas para el Adelanto de la Mujer, tales como: ${ }^{22}$

1. El establecimiento de mecanismos adecuados y fiables para controlar los progresos realizados.

2. La identificación de las cuestiones y problemas en todas las áreas de actividad que permita diagnosticar las diferencias y disparidades en razón del género.

3. La realización sistemática del análisis por géneros.

4. Contar con una clara voluntad política y la asignación de los recursos adecuados, incluidos recursos adicio-

21. Esta comisión forma parte del Consejo Económico y Social de las Naciones Unidas, como un órgano intergubernamental mundial dedicado exclusivamente a la promoción de la igualdad de género y el empoderamiento de las mujeres. Naciones Unidas. (2019). Manual básico sobre la Agenda 2030 para el Desarrollo Sostenible.

22. Organización Internacional del Trabajo. (s/f). Instrumentos para la igualdad de género. https://www.ilo.org/public/spanish/bureau/gender/newsite2002/about/defin. htm\#: :text=\%22Transversalizar\%20la\%20perspectiva\%20de\%20g\%C3\%A9nero,y\%20en\%20todos\%20los\%20niveles. 
nales financieros y humanos para traducir la idea de transversalización de género en realidad.

5. No eliminar la necesidad de elaborar políticas y programas específicamente destinados a las mujeres, así como una legislación positiva en su favor; y jamás dar por supuesto que hay cuestiones o problemas indiferentes desde la perspectiva de la igualdad entre los géneros.

Para avanzar hacia la incorporación de la perspectiva de género es necesario trabajar en la generación de indicadores y estadísticas en la materia que nos permitan ver las áreas de menor participación de la mujer y las que más le afectan. La falta de indicadores claros es un gran obstáculo para medir el avance de la incorporación de la perspectiva de género en los procesos nacionales y para evaluar con claridad las acciones que todavía se requieren para lograr la plena incorporación de la mujer en los diversos ámbitos sociales, políticos, económicos y culturales. ${ }^{23}$ Según los datos disponibles, para dar seguimiento a las cuestiones de género a nivel mundial se cuenta con indicadores y datos que representan apenas el $26 \%{ }^{24}$

Si pretendemos incorporar a la mujer plenamente en todas las esferas de la sociedad y alcanzar una equidad de género en cumplimiento con los ODS, es imprescindible contar con datos segregados fiables y de calidad, que incluyan datos desglosados por ingresos, sexo, edad, raza, origen étnico, estatus migratorio, discapacidad, ubicación geográfica, etc., y que pongan de realce la situación de los grupos más vulnerables. Para evitar este tipo de restricción en los datos y de acceso a la información, se requiere que se inte-

23. Maldonado Mera, Betzabé, Lara Burbano, Giovanna, y Maya Carrillo,Azucena. (2016). La mujer como motor del desarrollo local: Una experiencia puntual (p. 93).

24. Onu Mujeres. (2018). Hacer las promesas realidad: La igualdad de género en la Agenda 2030 para el Desarrollo Sostenible (p. 48). 
gre una perspectiva de género en los programas nacionales de estadística de cada país y se priorice la recopilación de datos en tiempo y forma.

Para ello es importante empezar analizando la incorporación de la perspectiva de género en cada uno de los oDs. Si hacemos un análisis de los oDs podemos observar que las cuestiones de género se encuentran presentes en $11 \mathrm{de}$ los 17 Objetivos. En la siguiente gráfica podemos observar claramente el porcentaje que representan los indicadores que tiene una relación directa con las cuestiones de género en los 17 oDs.

\section{Gráfica 1}

\section{Porcentaje de inclusión de la perspectiva de género en los ODS (\%)}

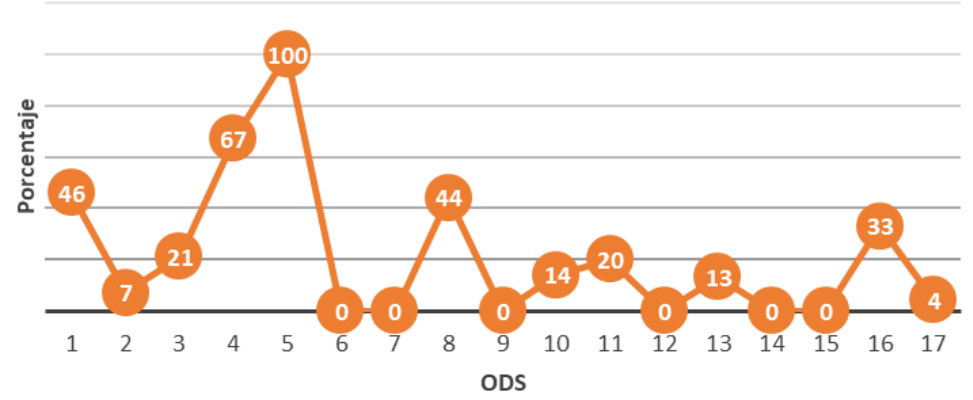

Fuente: elaboración propia con información obtenida en onu Mujeres (2018), y en a/res/71/313 Marco de indicadores mundiales para los Objetivos de Desarrollo Sostenible y metas de la Agenda 2030 para el Desarrollo Sostenible.

De los 248 indicadores analizados podemos observar que sólo 57 de éstos abordan temas relativos a las mujeres o cuestiones de género. Es decir, del total de los indicadores contenidos en las 169 metas de los 17 oDs, sólo el $22 \%$ tiene una relación directa con cuestiones de género. Asimismo, es 
necesario precisar que de los 57 indicadores con perspectiva de género contenidos en los oDs, sólo 10 indicadores proporcionan información suficiente y periódica.

Como era de esperarse, el oDs 5 es el que cuenta con el $100 \%$ de los indicadores relacionados con las cuestiones de género, es decir los 14 indicadores contenidos en este Objetivo tienen una relación con las cuestiones de género. Seguido del oDs 4 que cuenta con ocho indicadores relacionados con las cuestiones de género de los 12 indicadores totales, es decir tiene un porcentaje del $67 \%$ de estos indicadores; el oDs 1 cuenta con seis de los 13 indicadores, representando $46 \%$ de incorporación de la perspectiva de género; el oDs 8 cuenta con siete de los 16 indicadores contenidos en este Objetivo, para 44\% de incorporación de perspectiva de género; el oDs 16 cuenta con ocho de los 24 indicadores, $33 \%$ de perspectiva de género, y oDs 3 cuenta con seis indicadores con perspectiva de género de los 28 contenidos en este Objetivo, para un 21\%. En menor medida podemos encontrar la perspectiva de género en los indicadores contenidos en el oDs 11, con tres indicadores de género de los 15 contenidos en este objetivo, para 20\%; el oDs 10 dos indicadores de los 14 totales, o 14\%; el oDs 13 un indicador de los ocho contenidos en este Objetivo que hace referencia a las cuestiones de género, para un 13\%; el oDs 2 un indicador de género de los 14 contenidos en este Objetivo, o 7\%, y el oDs 17 un indicador de género de los 24 totales contenidos en este Objetivo, para un $4 \%$. Por su parte, en los oDs relativos al agua limpia y saneamiento (6), el de energía asequible y no contaminante (7), el de industria, innovación e infraestructura (9), el de producción y consumo responsables (12), el de vida marina (14) y el de vida de ecosistemas terrestres (15) no se encontró ningún indicador relacionado con las cuestiones de género. En las cuestiones ambientales, en las cuales curiosamente se ha identificado una falta de incorporación de indicadores de género, la importancia de 
la participación de las mujeres es fundamental, ya que son ellas quienes transmiten de generación en generación los conocimiento básicos del cuidado de los bosques, las tierras y el agua; quienes dedican muchas horas a la recolección de leña, forraje y agua para la limpieza de sus hogares y la preparación de alimentos; y quienes suelen ser las primeras afectadas por los desastres naturales y en sufrir las consecuencias económicas y sociales que eso trae consigo.

\section{Promoción de una cultura de desarrollo sostenible con perspectiva de género en los ODS}

El desarrollo humano, como bien señalan Martínez de Soria y Carrica Ochoa, es el crecimiento o mejora del ser humano que sirve de orientación para promover cambios en los procesos sociales que propicien bienestar y una mayor calidad de vida. ${ }^{25}$ Desde los años noventa el desarrollo se empezó a medir bajo el Índice de desarrollo humano que incluye indicadores tales como los ingresos básicos, la esperanza de vida al nacer, el alfabetismo, etc. A partir de entonces el concepto se ha ido ampliando hasta incluir aspectos relacionados con las oportunidades, más que con las cuestiones económicas de las personas. Teniendo como fin último la libertad plena del ser humano, sin condicionamientos que le impidan su crecimiento personal, profesional, económico y social. Autores como Amartya Sen y Martha Nussbaum han trabajado bastante en incorporar nuevos elementos que pudieran impedir el logro del bienestar y desarrollo humano. Sostienen que las políticas deben orientarse hacia el logro del bien para el ser humano y sobre todo al reforzamiento de las capacidades de los individuos.

En su libro Las mujeres y el desarrollo humano. Enfoque de las capacidades, Martha Nussbaum hace referencia a 
la importancia que tiene la mujer en el desarrollo y resalta cómo las condiciones precarias a las que se ven sometidas muchas mujeres alrededor del mundo condicionan su acceso a una mejor calidad de vida. Estas situaciones políticas y sociales, comenta, generan desigualdades en las capacidades humanas, convirtiendo a las mujeres, más que en un fin en sí mismas, en meros instrumentos para los fines de otros. ${ }^{26}$

En línea con lo que señalan Maldonado et al., el nuevo modelo de desarrollo humano busca que la persona se convierta en protagonista de su propio progreso; para ello, señala, es indispensable fortalecer las capacidades, impulsar los emprendimientos productivos que mejoren paulatinamente la calidad de vida, de las familias y la comunidad sin comprometer los recursos de las generaciones futuras. ${ }^{27}$

Para promover un verdadero desarrollo humano basado en la equidad, es imprescindible trabajar en la promoción de los derechos humanos en general y especialmente en los derechos de las mujeres y las niñas, que promuevan la construcción de una cultura de igualdad de género y derechos humanos.

Numerosas investigaciones apoyan el aporte de las mujeres en el desarrollo económico pese a las dificultades a las que se enfrentan. Según señalan Maldonado et al., las primeras investigaciones sobre esta cuestión las podemos encontrar en el año 1930, pero no es hasta 1970 cuando se crea un punto de inflexión gracias al estudio desarrollado por Ester Boseup al poner en evidencia que el desarrollo no es un neutro o indiferente a las cuestiones de género, abriendo con ello el debate teórico político sobre las cuestiones de género en el desarrollo. ${ }^{28}$ Este estudio, según señalan Maldonado et al., se apoya en tres grandes teorías: que

26. Nussbaum, Martha. (20I2). Las mujeres y el desarrollo humano. El enfoque de las capacidades.

27. Maldonado Mera, Betzabé, Lara Burbano, Giovanna, y Maya Carrillo,Azucena. (2016). La mujer como motor del desarrollo local: Una experiencia puntual (p. 95). 28. Ibíd., p. 96.

Estado No.82 
existe un sesgo de género implícito en los tres niveles en lo que se desarrolla la economía (micro, meso y macro); que se debe considerar que existe una interdependencia entre las esferas de la economía productiva y reproductiva para mantener el equilibro entre la satisfacción de las necesidades básicas del ser humano, su familia y su comunidad; y que las tareas domésticas y la economía del cuidado implican la producción de bienes, servicios, actividades y valores que generan valor y por tanto son parte del flujo económico de las sociedades. ${ }^{29}$ Así, estas acciones refuerzan la idea del valor integral que tiene la mujer en la construcción de desarrollo.

Como podemos observar, a través de los derechos humanos se fomenta la construcción de sociedades más justas y equitativas, mientras que a través de la cultura se promueve y facilita la incorporación de los cambios positivos en los patrones tradicionales de conducta. En ambas cuestiones la participación de la mujer es fundamental, ya que es a través de ella desde donde se promueven y transmiten los valores culturales como la transmisión de la lengua, los usos y costumbres, la elaboración y comprensión cultural en la vestimenta, etc.; y desde la cual en la mayoría de las veces se reproducen patrones sociales que facilitan y/u obstaculizan la promoción de los derechos humanos. ${ }^{30}$

Como se ha puntualizado desde la UnEsco, la igualdad de género en la cultura no es inmune a las desigualdades, ya que la discriminación se extiende a otras áreas de la sociedad y recibe la influencia de un contexto más amplio que se incluye en otras formas de categorización social como la religión, la discapacidad o los niveles de pobreza, que pueden conllevar grandes desventajas. ${ }^{31}$

29. Ibíd., p. 97.

30. Unesco. (2015). Igualdad de género. Patrimonio y creatividad. Centro Internacional para la Promoción de los Derechos Humanos.

3l. Ibíd., p. 14. 
Es importante resaltar que la igualdad de género está siendo reconocida ampliamente como un objetivo principal del desarrollo humano. ${ }^{32}$ La Convención sobre la Eliminación de Todas las Formas de Discriminación Contra la Mujer (1979) enfatiza en su Preámbulo que

[...] la máxima participación de la mujer en todas las esferas, en igualdad de condiciones con el hombre, es indispensable para el desarrollo pleno y completo de un país, el bienestar del mundo y la causa de la paz, [y que] el establecimiento del nuevo orden económico internacional basado en la equidad y la justicia contribuirá significativamente a la promoción de la igualdad entre el hombre y la mujer. ${ }^{33}$

En la Resolución 68/223 de la Asamblea General de las Naciones Unidas se resalta el papel integral que tiene la promoción de la igualdad de género en la obtención de un desarrollo sostenible a través de la cultura, instando a los Estados en su artículo 11c a

[...] asegurar que las mujeres y los hombres tuvieran igual acceso, participación y contribución a la vida cultural y a la toma de decisiones, además de comprometerse al desarrollo de políticas y programas culturales con perspectiva de género, en los ámbitos locales, nacionales e internacionales a modo de promover la igualdad de género y el empoderamiento de mujeres y niñas. ${ }^{34}$

Debemos recordar que en muchos países alrededor del mundo se siguen llevando a cabo prácticas discriminatorias

32. Asamblea General de las Naciones Unidas. (2019). Estudio mundial sobre el papel de la mujer en el desarrollo. Informe del secretario general. La importancia de hacer frente a la pobreza económica y la pobreza de tiempo de las mujeres en favor del desarrollo sostenible.

33. Asamblea General de las Naciones Unidas. (1979). Convención sobre la eliminación de todas las formas de discriminación contra la mujer (p. I).

34. Asamblea General de las Naciones Unidas. (2013). Resolución 68/223. Cultura y desarrollo sostenible. a/res/68/223, p. 5. 
que transgreden o violan los derechos de las mujeres y las niñas, tales como la violencia doméstica, el matrimonio precoz o la mutilación genital femenina (MGF), entre otras. Lo que, según la UNESCO, ha llevado a una errónea y desafortunada combinación entre prácticas tradicionales perjudiciales y "cultura", justificando dichas acciones como parte de la cultura y poniendo en un segundo plano los derechos humanos, especialmente los derechos de las mujeres y las niñas. ${ }^{35}$ Ante esta situación debemos tener claro que el respeto por la dignidad humana debe estar por encima de las prácticas tradicionales perjudiciales. En este sentido, la Convención señala en su artículo $5^{\circ}$ y en su preámbulo que es necesario

[...] modificar los patrones socioculturales de conducta de hombres y mujeres, con miras a alcanzar la eliminación de los prejuicios y las prácticas consuetudinarias y de cualquier otra índole que estén basados en la idea de la inferioridad o superioridad de cualquiera de los sexos o en funciones estereotipadas de hombres y mujeres, [ya que] para lograr la plena igualdad entre el hombre y la mujer es necesario modificar el papel tradicional tanto del hombre como de la mujer en la sociedad y en la familia. ${ }^{36}$

Los informes del Fondo de Población de las Naciones Unidas (UNFPA, 2008) señalan que las desigualdades de género están insertas en muchas instituciones de las sociedades, desde la familia hasta el Estado. ${ }^{37}$ Por ello es importante mantener una visión crítica y analítica que permita detectar las situaciones que afectan diariamente a las mujeres alrededor del mundo. En este sentido, la unEsCo ha elaborado una

35. Unesco. (2015). Igualdad de género. Patrimonio y creatividad. Centro Internacional para la Promoción de los Derechos Humanos.

36. Asamblea General de las Naciones Unidas. (1979). Convención sobre la eliminación de todas las formas de discriminación contra la mujer (p. 2-4).

37. Ídem. 
serie de recomendaciones para incorporar la perspectiva de género en el desarrollo.

Recomendaciones de la UNESCO para incorporación de la perspectiva de generó en el desarrollo: ${ }^{38}$

1. Asegurar la plena aplicación de las convenciones y declaraciones internacionales en el campo de la cultura en consonancia con otros instrumentos de derechos humanos y respecto a la igualdad de género y diversidad para ampliar los horizontes creativos de mujeres y hombres, niños y niñas, y para garantizar el acceso y la participación equitativa de la vida cultural.

2. Fortalecer el sustrato de pruebas por medio de la recopilación y difusión periódica y sistemática por parte de oficinas nacionales de estadística, de datos clasificados por sexo en todas las áreas del sector cultural, incluso el empleo, la educación, el fortalecimiento de las capacidades, la participación y el consumo.

3. Desarrollar y aplicar políticas con perspectiva de género y estrategias en cultura que empoderen a todos los miembros de la sociedad, considerando la diversidad de los distintos grupos y personas y la intersección de factores sociales más amplios y desigualdades que pueden llevan a mayores desventajas.

4. Reforzar las capacidades institucionales nacionales para promover el acceso equitativo de mujeres y hombres a los procesos de toma de decisiones, recursos financieros y educación en el campo de la cultura.

5. Establecer iniciativas de liderazgo y orientación para creadoras y profesiones de patrimonio, y asegurar un equilibrio del género en los puestos de liderazgo en el sector cultural y creativo.

6. Respaldar las campañas internacionales, nacionales y locales de sensibilización y de promoción que abordan

38. Unesco. (20I5). Igualdad de género. Patrimonio y creatividad. Centro Internacional para la Promoción de los Derechos Humanos, p. I 35. 
los estereotipos de género y la discriminación en todos los aspectos de la vida cultural.

7. Estimular e involucrar a todos los miembros de la sociedad en estrategias que promuevan la igualdad de género en la cultura. Esto incluye trabajar en cooperación con todos los grupos y comunidades interesadas en promover soluciones sostenibles para un acceso en el que se respete la igualdad de género a la cultura, participación y contribución a ella.

8. Apoyar la investigación multidisciplinaria sobre igualdad de género en el patrimonio y las industrias creativas que involucran a los grupos y comunidades interesados, y que tienen en consideración la complejidad y diversidad de las relaciones de género y las estructuras de poder subyacentes.

Con estas recomendaciones, aunadas a las que se promueven desde onU Mujeres o desde los ods en la Agenda 2030, confiamos que se puede avanzar hacia la construcción de una cultura basada en la igualdad de género y el respeto de los derechos humanos como elemento clave para la generación de desarrollo sostenible.

De acuerdo con lo que señala Marcela Lagarde, para construir un desarrollo humano sostenible y viable es necesario no sólo enfrentar la privación humana y la depredación social, cultural y ambiental, sino reconocer y preservar la diversidad para mejorar la calidad de vida y generar mayores posibilidades de desarrollo personal y comunitario. ${ }^{39}$

Por su parte, la Guía de género de la Agencia Española de Cooperación al Desarrollo (AECID) señala que si pretendemos trabajar en el diseño de estrategias para la inclusión de la perspectiva de género en el desarrollo, es imprescindible tomar en consideración que:

39. Lagarde, M. (1996). Género y feminismo. Desarrollo humano y democracia (pp. 10 y II). 
[...] la igualdad entre mujeres y hombres está en la base del desarrollo; que existe una situación de desigualdad social entre mujeres y hombres respecto a las actividades que desempeñan, a su acceso a recursos y el control de los mismos y a su participación en la toma de decisiones; que las estrategias de desarrollo no son neutras en relación con el género, al tener consecuencias positivas y negativas diferenciadas para hombres y mujeres; que las acciones de desarrollo son más eficaces si tienen en cuenta las diferencias y desigualdades entre mujeres y hombres; que la invisibilización de las mujeres en las intervenciones de desarrollo complica la definición de las estrategias para apoyar la satisfacción de las necesidades prácticas e intereses estratégicos; que analizar los contextos y la realidad desde el género implica no considerar a todas la mujeres y hombres como iguales; que trabajar con mujeres no significa que automáticamente se tienen en cuenta los aspectos relacionados con la desigualdad de género desde un enfoque de derechos humanos, pudiéndose impulsar proyectos exclusivos para mujeres que tengan un efecto muy negativo en las relaciones de género y refuercen su posición de subordinación; que los hombres son un elemento clave para el cambio de las relaciones de género. Es imprescindible trabajar con ellos para eliminar las causas estructurales de la desigualdad de género y modificar la percepción "tradicional" que tanto hombres como mujeres tienen en su "rol" de género; que los esfuerzos para corregir las desigualdades a través de proyectos dirigidos a mujeres pueden resultar contraproducentes si los hombres no son informados e incluidos a lo largo del proceso; $y$ finalmente que las mujeres no son un grupo vulnerable (representan más de la mitad de la población mundial) pero debido a las múltiples discriminaciones de las que son objeto están en condiciones de exclusión y a veces mayor vulnerabilidad. ${ }^{40}$

En línea con lo que señala esta Guía y con lo que se manifiesta en la Agenda 2030 acerca de que "el desarrollo sólo será sostenible si los beneficios favorecen por igual a muje-

40. Aecid. (20I5). Guía de la aecid para la transversalización del enfoque de género (p. I5).

Estado No. 82 
res y hombres", ${ }^{41}$ podemos vislumbrar el papel fundamental que desempeña la mujer en la generación de desarrollo; en la formación integral de cada uno de los miembros de su núcleo familiar en valores clave para la construcción de sociedades más justas, equitativas y solidarias; en la gestión de los recursos económicos y materiales del hogar; y en su participación activa en el mercado laboral. ${ }^{42}$

Como ya hemos hecho mención, dentro de la Agenda 2030 existen seis oDs que no incluyen en ninguna de sus metas e indicadores la perspectiva de género. Esto limita la incorporación de dicha perspectiva tanto de los gobiernos como de las organizaciones de la sociedad civil en el cumplimiento de los oDs en las acciones relacionadas con el cuidado del medio ambiente y de ciudades seguras y sostenibles.

Según onu Mujeres siguen existiendo grandes retos para la incorporación del enfoque de género en los oDs,$^{43}$ entre los que se encuentran la falta de elaboración de indicadores claros y específicos de género en los oDs, ya que existen un gran número de estos Objetivos que no cuenta con ningún indicador sobre género, y de los que cuentan con indicadores de género la información obtenida sigue siendo insuficiente. La falta de datos, como bien señalan Maldonado Mera et $a l$. , ha provocado que no se incorpore a las estadísticas de muchos países el importante aporte que ha tenido la mujer en el desarrollo económico de los pueblos. ${ }^{44}$ Otro de los retos, según señala este organismo, es la incorporación transversal de la perspectiva de género en todas las políticas públicas diseñadas para el cumplimiento de los oDs, proponiendo que

4I. Onu Mujeres. (2018). Hacer las promesas realidad. La igualdad de género en la Agenda 2030 para el Desarrollo Sostenible (p. I).

42. Maldonado Mera, Betzabé, Lara Burbano, Giovanna, y Maya Carrillo,Azucena. (2016). La mujer como motor del desarrollo local: Una experiencia puntual (p. 93).

43. Onu Mujeres. (2018). Hacer las promesas realidad: la igualdad de género en la Agenda 2030 para el Desarrollo Sostenible (p. 68).

44. Ídem. 
se trabaje en la formación de los funcionarios públicos para la implementación del enfoque en las políticas nacionales.

Si bien podemos observar la valiosa aportación de la mujer en las estrategias para la generación de desarrollo sostenible, su participación sigue siendo limitada y poco valorada. La falta de indicadores claros y específicos de género, como ya hemos hecho mención, limita no sólo la visibilización y el reconocimiento de las acciones de las mujeres en la generación de desarrollo, sino que continúa perpetuando prácticas discriminatorias contra la mujer. Por ello es importante que se continúe trabajando en la elaboración de indicadores que pueden medir la participación de la mujer en cada uno de las esferas que abarcan los 17 ODS, para reconocer y visibilizar la importancia que tiene la misma en el cumplimiento de los oDs y por ende en la generación de desarrollo sostenible.

\section{Conclusiones}

Son innegables los grandes avances que se han venido produciendo en los últimos 50 años en la promoción de los derechos de la mujer y su participación activa e igualitaria en los diversos sectores de la sociedad. Sin embargo, todavía siguen existiendo enormes obstáculos políticos, sociales, económicos y culturales que impiden el reconocimiento de sus derechos y por ende su participación plena en los diversos ámbitos de la sociedad.

Las prácticas tradicionales discriminatorias que se siguen realizando en algunos países alrededor del mundo, tales como la violencia doméstica, el matrimonio precoz o la mutilación genital femenina (MGF), entre otras, violan o transgreden los derechos de numerosas mujeres y niñas. El hecho de ser parte de las tradiciones culturales de algunos pueblos no justifica que ponga en segundo plano los derechos de las mujeres y las niñas. En ese sentido, debemos tener 
claro que el respeto por la dignidad humana debe estar por encima de las prácticas tradicionales perjudiciales.

Para ello, algunos organismos internacionales como ONU Mujeres, la unesco o la Agencia Española de Cooperación para el Desarrollo (AECID) han elaborado algunas recomendaciones para incorporar la perspectiva de género tanto en las políticas nacionales como en las acciones destinadas a la cooperación al desarrollo, como en la construcción de una cultura basada en el respeto de los derechos humanos y especialmente de igualdad de género. Dichas acciones se resumen en la elaboración de indicadores claros y específicos de género en las políticas nacionales destinadas hacia el cumplimiento de los oDs; la incorporación de los hombres en las acciones y programas de igualdad de género; la formación de funcionarios públicos en cuestiones de igualdad de género; en la promoción y fomento de la participación de todos los actores de la sociedad en las acciones y programas destinados a la creación de una cultura basada en la igualdad de género y en el respeto de los derechos humanos, así como en la elaboración de campañas que fomenten la sensibilización de la sociedad, la eliminación de los estereotipos de género y las prácticas discriminatorias por cuestiones de género, entre otros.

Por su parte, desde la Agenda 2030 para el Desarrollo Sostenible se ha trabajado en la incorporación de la perspectiva de género en las metas e indicadores contenidos en los oDs. Como bien hemos señalado anteriormente, todavía queda mucho trabajo por hacer ya que de los 17 oDs, seis Objetivos no hacen ninguna referencia a las cuestiones de género $(6,7,9,12,14$ y 15). En menor medida encontramos la perspectiva de género en cinco oDs $(2,10,11,13$ y 17) y en mayor medida encontramos dicha perspectiva en seis ods (1, 3, 4, 5, 8 y 16). En todos ellos la participación de la mujer es imprescindible, ya que si queremos que la mujer logre empoderarse mediante el acceso a recursos econó- 
micos que le permitan mantener su hogar y su entorno en condiciones óptimas y saludables, debemos trabajar en los oDs $1,6,7,11,12,13,14$ y 15; para poder alimentarse adecuadamente y alimentar y proporcionar una educación de calidad a los miembros de su hogar debemos fomentar su participación activa en los oDs 2 y 4; para cuidar su salud y la de los suyos y velar por la seguridad física, emocional y económica personal y familiar debemos reforzar los ODS 3 y 16. Con todo ello podríamos contribuir al cumplimiento de las metas e indicadores contenidos en la Agenda 2030, y con ello mejorar la vida de muchas mujeres y sus familias alrededor del mundo.

Si tomamos en consideración que bajo el nuevo modelo de desarrollo cada persona es la protagonista de su propio desarrollo, debemos entonces proporcionar a cada persona las herramientas claves tanto económicas como políticas, sociales y culturales que le permitan alcanzar dicho desarrollo. Sin embargo, sabemos que el desarrollo humano sigue siendo inequitativo y desigual y lo seguirá siendo si seguimos negando o invisibilizando la valiosa labor que tiene la mujer en la construcción de un desarrollo humano sostenible.

AECID. (20I5). Guía de la AECID para la transversalización del Bibliografía enfoque de género. Madrid, España:Agencia Española de la Cooperación Internacional para el Desarrollo.

Asamblea General de las Naciones Unidas. (1979). Convención sobre la Eliminación de Todas las Formas de Discriminación Contra la Mujer. Resolución 34//80, de 18 de diciembre de 1979.

—_. (2013). Resolución 68/223. Cultura y desarrollo sostenible. A/RES/68/223, p. 7.

—. (20I5). Transformar nuestro mundo: La Agenda 2030 para el Desarrollo Sostenible. Resolución 70/l, 25 de septiembre de 20I5, A/RES/70/I. 
Bibliografía
- (2019). Estudio mundial sobre el papel de la mujer en el desarrollo. Informe del secretario general. La importancia de hacer frente a la pobreza económica y la pobreza de tiempo de las mujeres en favor del desarrollo sostenible ( $\mathrm{p}$. I I7), I7 de junio de 2019,A/74/I I I.

Bernal Martínez de Soria, Aurora, y Carrica Ochoa, Sarah. (2014). Mujer, desarrollo y educación para el desarrollo. Edetania, núm. 46, pp. I8I-199. Universidad de Navarra-Facultad de Educación y Psicología.

Calatrava, Javier. (2002). Mujer y desarrollo rural en la globalización: De los proyectos asistenciales a la planificación de género. Globalización y Mundo Rural, núm. 803, p. 73. Fernández Saavedra,Ana G.,y Dema Moreno, Sandra. (2018). La integración de la perspectiva de género en la gestión del riesgo de desastres: De los ODM a los ODs. Revista Internacional de Cooperación y Desarrollo, 5(I): 3 I-43.

Fundación Mujeres. (2003). Guía para la elaboración de proyectos desde una perspectiva de género (p. 43). Fondo Social Europeo/Gobierno del Principado de Asturias.

Grupo de las Naciones Unidas para el Desarrollo Sostenible. (2019). Marco de Cooperación de las Naciones Unidas para el Desarrollo Sostenible. Directrices internas (p. 38). Naciones Unidas.

Lagarde, Marcela. ( 1996). Género y feminismo. Desarrollo humano y democracia. Horas Horas, p. 248. Editorial Madrid.

Machado López, L., Morales Molina, T., y Chávez Calle, L. S. (2018). La igualdad de género, paradigma del desarrollo sostenible en laAgenda 2030. Universidad y Sociedad, I0(2): 7-13. Recuperado de http://rus.ucf.edu.cu/index.php/rus Maldonado Mera, Betzabé, Lara Burbano, Giovanna, y Maya Carrillo, Azucena. (2016). La mujer como motor del desarrollo local: Una experiencia puntual. Revista San Gregorio, número especial sobre desarrollo local, pp. 92-107. 
Ministerio de Asuntos Exteriores y de Cooperación. (2007). Estrategia de "género en desarrollo" de la cooperación española. Secretaría de Estado de Cooperación Internacional-Dirección General de Planificación y Evaluación de Políticas para el Desarrollo.

Nussbaum, Martha. (2012). Las mujeres y el desarrollo humano. El enfoque de las capacidades. Herder Editorial.

ONu Mujeres. (20I2). un System-wide action plan for implementation of the CEB United Nations System wide policy on Gender Equal and the empowerment of women. https://www.unwomen.org/-/media/headquarters/ attachments/sections/how\%20we\%20work/unsystemcoordination/un-swap/un-swap-framework-dec-20I 2. pdf? la $=$ es\&vs $=1815$

- (2018). Hacer las promesas realidad: La igualdad de género en la Agenda 2030 para el Desarrollo Sostenible. Estados Unidos.

Organización de las Naciones Unidas (ONU). (2019). Manual básico sobre la Agenda 2030 para el Desarrollo Sostenible. Uruguay: ONU.

UNESCO. (20I5). Igualdad de género. Patrimonio y creatividad. UnESCO-Centro Internacional para la Promoción de los Derechos Humanos. http://www.unesco.org/culture/ Gender-Equality-and-Culture/flipbook/es/mobile/index. html\#p=2

Verdiales López, D. (2020). La importancia de la mujer en el desarrollo. Análisis de los Objetivos de Desarrollo Sostenible con perspectiva de género. FEMERIS. Revista Multidisciplinar de Estudios de Género, 5(3): 97-I I 3, noviembre. Zabala Errazti, I., Martínez Herrero, M. J., y Labaien Egiguren, I.(2012).Análisis de la integración del enfoque de género en las políticas de cooperación al desarrollo: El caso de las instituciones de la Comunidad Autónoma del País Vasco. Estudios de Economía Aplicada, 30(3): 94I-970. 